\title{
A Two Band Model for Superconductivity: Probing Interband Pair Formation
}

\author{
R. E. Lagos $^{a)}$ and G. G. Cabrera ${ }^{b)}$ \\ ${ }^{a)}$ Departamento de Física, IGCE, Universidade Estadual Paulista (UNESP) \\ CP. 178, 13500-970, Rio Claro, SP, Brazil

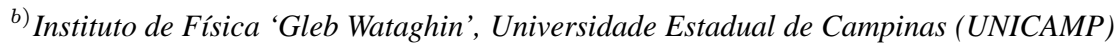 \\ CP. 6165 Campinas, SP 13083-970 Brazil
}

Received on 23 May, 2003

\begin{abstract}
We propose a two band model for superconductivity. It turns out that the simplest nontrivial case considers solely interband scattering, and both bands can be modeled as symmetric (around the Fermi level) and flat, thus each band is completely characterized by its half-band width $W_{n}(\mathrm{n}=1,2)$. A useful dimensionless parameter is $\delta$, proportional to $W_{2}-W_{1}$. The case $\delta=0$ retrieves the conventional BCS model. We probe the specific heat, the ratio gap over critical temperature, the thermodynamic critical field and tunneling conductance as functions of $\delta$ and temperature (from zero to $T_{c}$ ). We compare our results with experimental results for $M g B_{2}$ and good quantitative agreement is obtained, indicating the relevance of interband coupling. Work in progress also considers the inclusion of band hybridization and general interband as well as intra-band scattering mechanisms.
\end{abstract}

\section{Introduction}

Magnesium Diboride $\left(M g B_{2}\right)$ appears to be a rather "unconventional" conventional superconductor $[1,2]$. Two band effects observed as deviations of conventional BCS include: anomalous specific heat [3] and two gaps features (including double peaked tunneling conductance spectra) [410]. The superconductive mechanism, nevertheless seems to be conventional phonon BCS-like [11]. In this short communication we present a two band model based on the classical work by Suhl et al. [12] and on an extension of the latter applied to high $T_{c}$ compounds [13]. We mention other multiband models in the literature [14-21], and some calculations and fittings within a multiband and strong coupling context include Ref. [22-25]. In section II we introduce a two band model $[12,13]$ and within the usual BCS scheme we compute the mean field expressions for the free energy, entropy, critical field, conductance, and the selfconsistent equations for the gaps functions. In particular we consider the simplest case: solely interband pairing coupling via phonons. In section III we compare our simple model with some experimental results for the case of $M g B_{2}$ $[26,1,27]$, indicating that the interband pairing mechanism is somehow relevant. Finally in section IV we present some concluding remarks and future work.

\section{The two band model}

Our model follows Ref. [12, 13], with the Hamiltonian

$$
\begin{aligned}
H & =\sum_{\mathbf{k}, m} E_{k, m}\left(c_{k, m}^{\dagger} c_{k, m}-c_{-k, m} c_{-k, m}^{\dagger}\right) \\
& -\frac{1}{N} \sum_{\mathbf{k q}, m} V_{n, m} c_{k, n}^{\dagger} c_{-k, n}^{\dagger} c_{-q, m} c_{q, m}
\end{aligned}
$$

where the $c_{k}^{\dagger}$,s are the usual creation operators, $E_{k, m}$ are the bands dispersion ( $m=1,2), V_{n, m}$ are the positive pairing coefficients $\left(V_{12}=V_{21}\right.$ and $\left.D=V_{11} V_{22}-V_{12}^{2} \neq 0\right)$. We have defined $k=(\mathbf{k}, \uparrow),-k=(-\mathbf{k}, \downarrow), N$ is the number of sites and the last summation is with the usual energy cutoff $\omega_{D}$. The order parameters $\Delta_{n}$ are defined as the expectation values

$$
\Delta_{n}=\frac{1}{N} \sum_{\mathbf{k}, m} V_{n, m}\left\langle c_{k, m}^{\dagger} c_{-k, m}^{\dagger}\right\rangle
$$

The effective Hamiltonian is given by (within the Hartree Fock scheme for anomalous pairing, see Ref. [13])

$$
H_{\mathrm{eff}}=N E_{0}+\sum_{k, m} \Psi_{k, m}^{\dagger}\left(E_{k, m} \sigma_{z}-\Delta_{m} \sigma_{x}\right) \Psi_{k, m}
$$

where 


$$
E_{0}=\frac{1}{D}\left(V_{22} \Delta_{1}^{2}+V_{11} \Delta_{2}^{2}-2 V_{12} \Delta_{1} \Delta_{2}\right), \quad \Psi_{k, m} \equiv\left(\begin{array}{c}
c_{k, m} \\
c_{-k, m}^{\dagger}
\end{array}\right)
$$

and $\sigma_{x}, \sigma_{z}$ are the usual Pauli matrices. The free energy per site $F$ is given by

$$
\begin{aligned}
& \exp (-\beta N F)=\operatorname{Tr} \exp \left(-\beta H_{\mathrm{eff}}\right) \\
& F=E_{0}+\frac{T}{N} \sum_{k, m} \ln f_{k, m}\left(1-f_{k, m}\right)
\end{aligned}
$$

where $f(\omega)=(\exp (\beta \omega)+1)^{-1}, \omega_{k, m}=\sqrt{E_{k, m}^{2}+\Delta_{m}^{2}}$ and $f_{k, m}=f\left(\omega_{k, m}\right)$. The relative free energy $\delta F=$ $F-F\left(\Delta_{1}=\Delta_{2}=0\right)$, the thermodynamic critical field

$H_{c}$, entropy (per site) and specific heat are given, respectively by

$$
\delta F(T)=E_{0}-\frac{T}{N} \sum_{k, m} \ln \frac{\left(1+\cosh \beta \omega_{k, m}\right)}{\left(1+\cosh \beta E_{k, m}\right)}=-\frac{1}{8 \pi} H_{c}^{2}
$$

$$
S=-\frac{2}{N} \sum_{k, m}\left(\left(1-f_{k, m}\right) \ln \left(1-f_{k, m}\right)+f_{k, m} \ln f_{k, m}\right)
$$

The condensation energy is given by

$$
\delta F(T=0)=W_{C}=E_{0}-\frac{1}{N} \sum_{k, m}\left(\omega_{k, m}-E_{k, m}\right)
$$

and the superconductor- normal tunneling differential conductance (conveniently scaled) is defined by

$$
\begin{gathered}
G(V)=-\sum_{m} \int d \varepsilon \rho_{m, S}(\varepsilon) \frac{\partial f(\varepsilon+V)}{\partial \varepsilon} \\
\rho_{m, S}(\varepsilon)=\rho_{m}\left(\operatorname{sign}(\varepsilon) \sqrt{\varepsilon^{2}-\Delta_{m}^{2}}\right) \operatorname{Real}\left(\sqrt{\frac{(\varepsilon+i \Gamma)^{2}}{(\varepsilon+i \Gamma)^{2}-\Delta_{m}^{2}}}\right), \quad \Gamma \rightarrow 0^{+}
\end{gathered}
$$

Minimization of the free energy with respect to the gaps functions, yields a coupled nonlinear system of integral equations for the gaps, to be solved selfconsistently, and given by

$$
\begin{aligned}
\left(V_{22}-D R_{1}\left(\Delta_{1}, T\right)\right) \Delta_{1}-V_{12} \Delta_{2} & =0 \\
-V_{12} \Delta_{1}+\left(V_{11}-D R_{2}\left(\Delta_{2}, T\right)\right) \Delta_{2} & =0
\end{aligned}
$$

where

$$
R_{m}\left(\Delta_{m}, T\right)=\int_{-\omega_{D}}^{+\omega_{D}} d \varepsilon \rho_{m}(\varepsilon) S\left(\sqrt{\varepsilon^{2}+\Delta_{m}^{2}}\right), \quad S(x)=\frac{1}{2 x} \tanh \left(\frac{x}{2 T}\right)
$$

and with $\rho_{m}(\varepsilon)$ the density of states associated to the respective band. The transition temperature is the highest temperature $T_{c}=\beta_{c}^{-1}$, solution of

$$
\left(V_{22}-D R_{1}\left(0, T_{c}\right)\right)\left(V_{11}-D R_{2}\left(0, T_{c}\right)\right)=V_{12}^{2}
$$

\section{Results}

We compute the observables presented in the previous section. In particular we consider only interband scattering 
$V_{11}=V_{22}=0, V_{12}=\lambda$, the simplest relevant case $[12,13]$. We consider two flat symmetric bands, with $\rho_{m}(\varepsilon) \equiv \rho_{m}(0)=\rho_{m}$.

The gaps equations (7) now read

$$
\begin{gathered}
\Delta_{m}=\lambda \rho_{n} \Delta_{n} R\left(\Delta_{n}, T\right), \quad n \neq m=1,2 \\
R(\Delta, T)=\int_{0}^{\omega_{D}} \frac{d \varepsilon}{\sqrt{\varepsilon^{2}+\Delta^{2}}} \tanh \left(\frac{\beta}{2} \sqrt{\varepsilon^{2}+\Delta^{2}}\right)
\end{gathered}
$$

At zero temperature the gaps equations are given by (in conveniente units)

$$
\begin{aligned}
& \phi_{1}=\frac{2 \Delta_{1}(T=0)}{3.53 T_{c}}=\exp \frac{1}{\xi}(1-a) \\
& \phi_{2}=\frac{2 \Delta_{1}(T=0)}{3.53 T_{c}}=\exp \frac{1}{\xi}\left(1-\frac{1}{a}\right)
\end{aligned}
$$

where $\xi^{2}=\lambda^{2} \rho_{1} \rho_{2}$ and $a$ satisfies a selfconsistent equation. An excellent approximate solution is given by

$$
\ln a=\frac{\xi \theta}{2-\theta}, \quad \theta=\ln \sqrt{\frac{1+\delta}{1-\delta}}
$$

with

$$
-1<\delta=\frac{\rho_{1}-\rho_{2}}{\rho_{1}+\rho_{2}}<1
$$

Notice that all the above mentioned observables will yield the standard BCS expressions [28] in the limit $\delta=0$.

The critical temperature is given by $T_{c}=$ $1.13 \omega_{D} \exp \left(-\xi^{-1}\right)$.

We label the bands such that $\delta>0$. If we consider $M g B_{2}$, from Ref. [1, 27] we have $T_{c} \simeq 40{ }^{0} K, \omega_{D} \simeq$ $800^{\circ} K$ yielding $\xi \simeq 0.32$. From Ref. [26] we approximate $W_{1} \approx 5.6 \mathrm{eV}\left(\rho_{1} \approx 0.179 \mathrm{eV}^{-1}\right), W_{2} \approx 14 . \mathrm{eV}$ $\left(\rho_{2} \approx 0.071 \mathrm{eV}^{-1}\right)$ yielding $\delta \approx 0.432$.

In Fig. 1 we plot the normalized gaps $\phi_{m}$ at zero temperature, Eq.(8), and (minus) the condensation energy $-W_{c}$ Eq.(5), both as function of $\delta$. The condensation energy is normalized to the BCS reference state i.e. $W_{c}=\delta F(\delta, T=$ $0) / \delta F(\delta=0, T=0)$ (see Eq.2). The chosen normalization yields the standard BCS (weak coupling) value of unity for the gaps and the condensation energy. As $\delta$ is varied away from zero the condensation energy is less than the standard BCS. One gap will depart from weak to 'a medium coupling regime' $\left(\phi_{2}>1\right)$, conversely the other gap will dive towards 'a less than weak coupling regime' $\left(\phi_{1}<1\right)$, with the geometrical average $\sqrt{\phi_{1}(\delta) \phi_{2}(\delta)} \equiv 1$ always in the standard weak coupling regime. These features seem consistent as we fit the parameter $\delta$ with experimental data [1]-[10].

In order to solve for the gaps, Eq.(7), we can use the available low temperature and near the critical temperature expansions [28]. These allow us to nicely interpolate, for the full temperature regime $0 \leq \tau=T / T_{c} \leq 1$. Once this is done we can readily compute the specific heat, Eq.(4), entropy, Eq.(3), and the thermodynamic critical field, Eq.(2).

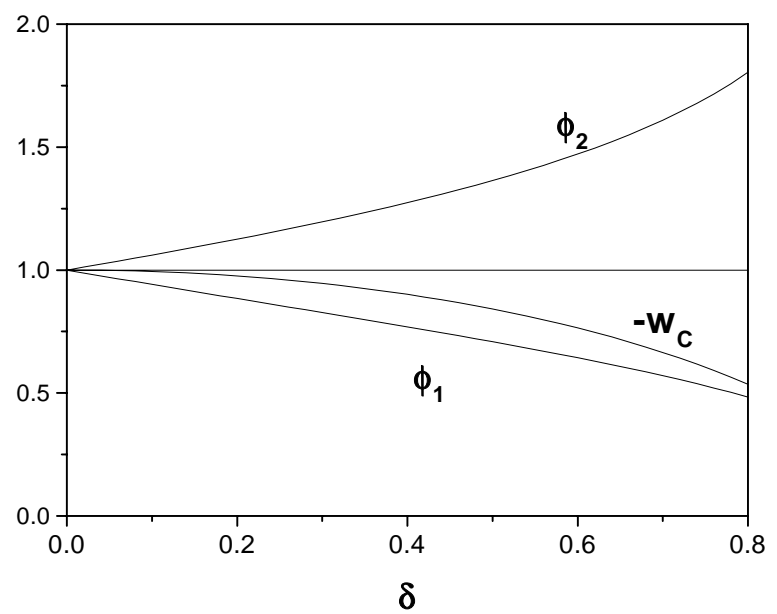

Figure 1. Gaps $\phi_{m}$ at zero temperature and (minus) the condensation energy $-W_{c}$ versus $\delta$. Convenient units $\phi_{m}=2 \Delta_{m}^{0} / 3.53 T_{c}$ and $W_{c}=\delta F(\delta, T=0) / \delta F(\delta=0, T=0)$. See text.

In Fig. 2 we plot the specific heat $C_{V}$ (normalized to the normal state value at $T_{c}$ ) versus the temperature $\tau$ for several values of $\delta$. The standard BCS result is represented by the curve $\delta=0$. The anomalous behavior of $C_{V}$ consists in going under the BCS value in the region $0.5<\tau<1$, and going over the BCS value in the region $0<\tau<0.5$. This feature is in very good agreement with Ref. [3]. In Fig. 3 we plot the entropy $S$ (normalized to the normal state value at $T_{c}$ ) versus the temperature $\tau$ for several values of $\delta$. The standard BCS result is represented by the curve $\delta=0$. As $\delta$ departs from zero (bands are less 'identical') the system increases its entropy. In Fig. 4 we plot the thermodynamic critical field (normalized to the reference state $\delta=0$, $T=0$ ) versus the temperature $\tau$ for several values of $\delta$. The standard BCS result is again represented by the curve $\delta=0$. As $\delta$ increases the critical field is reduced when compared to the BCS value. This is in agreement with experimental results [3].

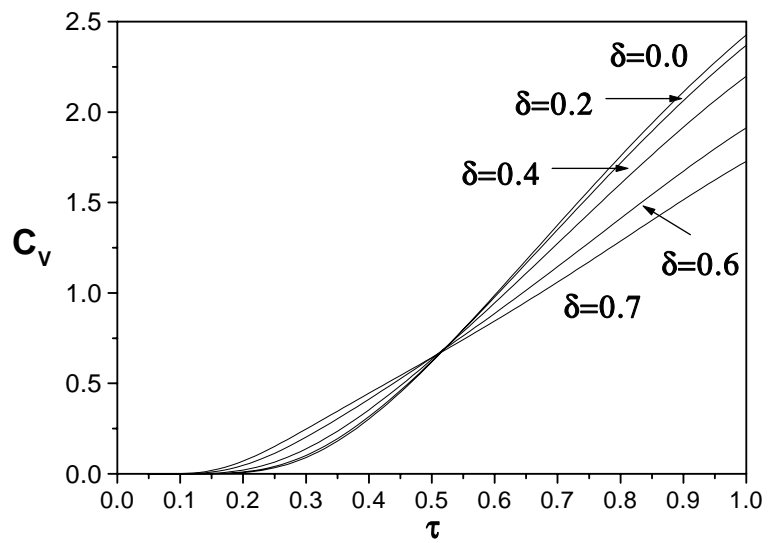

Figure 2. Specific heat $C_{V}$ versus $\tau=T / T_{c}$ for several $\delta$ values; normalized to the normal state specific heat at $T_{c}: C_{n}\left(T_{c}\right)=$ $4 \pi^{2}\left(\rho_{1}+\rho_{2}\right) / 6$. See text. 


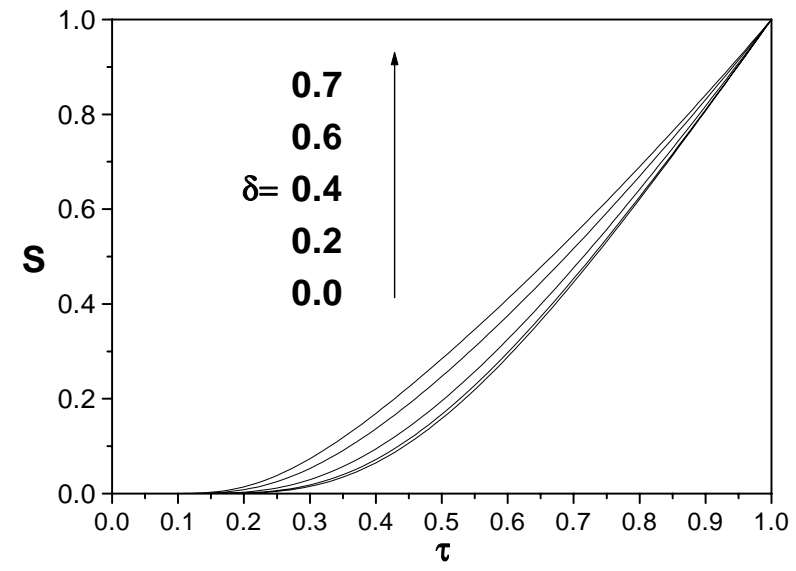

Figure 3. Entropy $S$ versus $\tau=T / T_{c}$ for several $\delta$ values; normalized to the normal state entropy at $T_{c}: S_{n}\left(T_{c}\right)=4 \pi^{2}\left(\rho_{1}+\right.$ $\left.\rho_{2}\right) T_{c} / 6$. See text.

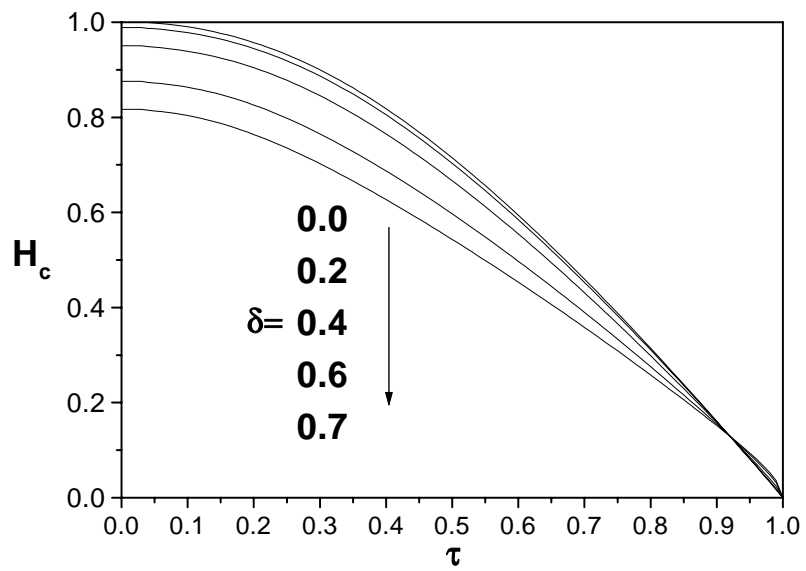

Figure 4. Thermodynamic critical field $H_{c}$ versus $\tau=T / T_{c}$ for several $\delta$ values; normalized to the reference (BCS) state $T=0$, $\delta=0, H_{c}^{2}(\tau)=F(\tau, \delta) / F(0,0)$. See text.

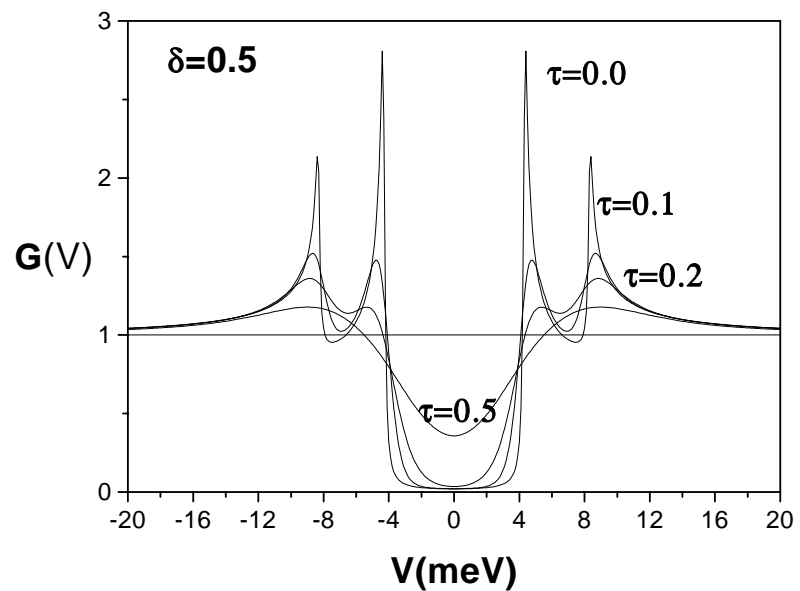

Figure 5. Tunneling conductance for several temperatures, at $\delta=0.5$, versus applied voltage. A small dispersion is included $\Gamma=0.1 \mathrm{meV}[22]$. See text.

In Fig. 5 we plot the conductance, Eq.(6) versus applied voltage, for a fixed value of $\delta=0.5$, and for several temperatures, and where a small dispersion is included, $\Gamma=0.1$
$\mathrm{meV}$ [22].The double peaked form is in very good agreement with observations (see for example Ref. [7]).

\section{Concluding Remarks}

We presented the simplest relevant two band model for superconductivity, based on a standard BCS-like pairing mechanism. We computed the gaps equations at zero temperature. Also the specific heat, entropy, critical field and conductance as function of temperature. We considered the simplest interband scattering mechanism (one pairing parameter) and two planar symmetrical bands (one parameter band model). Our results seems to be in very good agreement with some experimental results on the compound $M g B_{2}$, indicating that interband pairing is somehow relevant for this compound. These results are being investigated further. Work in progress incorporates intraband pairing mechanisms, an hybrid-like interband pairing mechanism [13], absent in most of the theoretical models, and a more involved band structure.

\section{References}

[1] C. Buzea and T. Yamashita; Supercond. Sci. Tech 14, R115 (2001).

[2] M. Angst and R. Puzniak; arXiv:cond-mat/0305048.

[3] F. Bouquet, R. A. Fisher, N. E. Phillips, D. G. Hinks, and J. D. Jorgensen; Phys. Rev. Lett. 87, 047001 (2001).

[4] S. Tsuda, T. Yokoya, T. Kiss, Y. Takano, K. Togano, H. Kito, H. Ihara, and S. Shin; Phys. Rev. Lett. 87, 177006 (2001).

[5] S. Tsuda, T. Yokoya, Y. Takano, H. Kito, A. Matsushita, F. Yin, H. Harima, and S. Shin; arXiv: cond-mat/0303636.

[6] G. Karapetrov, M. Iavarone, W. K. Kwok, G. W. Cabtree, and D. G. Hinks; Phys. Rev. Lett. 87, 4374 (2001).

[7] M. Iavarone, G. Karapetrov, A. E. Koshelev, W. K. Kwok, G. W. Cabtree, and D. G. Hinks; arXiv:cond-mat/0203329.

[8] A. Sharoni, I. Felner, and O. Millo; Phys. Rev. B63, 220508 (2001).

[9] H. Schmidt, J. F. Zasadzinski, K. E. Gray, and D. G. Hinks; Rev. B 63, 220504 (2001).

[10] Y. Bugoslavsky, Y. Miyoshi, G. K. Perkins, A. V. Berenov, Z. Lockman, J. L. MacManus-Driscoll, L. F. Cohen, A. D. Caplin, H. Y. Zhai, M. P. Paranthaman, H. M. Christen, and M. Blamire; Supercon. Sci. Tech. 15, 526 (2002).

[11] X. K. Chen, M. J. Konstantinović, J. C. Irwin, D. D. Lawrie, and J. P. Franck; Phys. Rev. Lett. 87, 157002 (2001).

[12] H. Suhl, B. T. Matthias, and L. R. Walker; Phys. Rev. Lett. 3, 552 (1959).

[13] R. E. Lagos, Solid State Commun. 79, 323 (1991).

[14] T. Örd and T. N. Kristoffel, Physica C370, 17 (2002).

[15] K. Yamaji; J. Phys. Soc. Jap. 59, 677 (1990), ibid. 701476 (2001).

[16] M. Imada; J. Phys. Soc. Jap. 70, 1218 (2001).

[17] N. Nakai, M. Ichioka, and K. Machida; J. Phys. Soc. Jpn. 71, 23 (2002). 
[18] J. E. Hirsch and F. Marsiglio, Phys. Rev. B 64, 144523 (2001).

[19] V. P. Ramunni, G. M. Japiassú, and A. Troper; Physica C364$365,190(2001)$

[20] I. N. Askerzade and A. Gencer; J. Phys. Soc. Jpn. 71, 1637 (2002)

[21] T. Mishonov and E. Penev; arXiv:cond-mat/0206118.

[22] H. J. Choi, D. Roundy, H. Sun, M. L. Cohen, and S. G. Louie; arXiv:cond-mat/0111183, Nature 418, 758 (2202).

[23] C. Joas, I. Eremin, D. Manske, and K. H. Bennemann; Phys. Rev. 65, 132518 (2002).
[24] A. A. Golubov, J. Kortus, O. V. Dolgov, O. Jepsen, Y. Kong, O. K. Andersen, B. J. Gibson, K. Ahn, and R. K. Kremer; J. Phys. Condens. Matter 14, 1353 (2002).

[25] A. Brinkman, A. A. Golubov, H. Rogalla, O. V. Dolgov, and J. Kortus; Phys. Rev. B65 180517 (2002).

[26] J. M. An and W. E. Pickett; Phys. Rev. Lett. 86, 4366 (2001).

[27] J. Kortus, I. I. Mazin, K. D. Belashchenko, V. P. Antropov, and L. L. Boyer; Phys. Rev. Lett. 86, 4656 (2001).

[28] M. Crisan; 'Theory of Superconductivity' World Scientific, 1989. 